\title{
Symmetrical and decoupled nickel microgyroscope on insulating substrate
}

\author{
Said Emre Alper, Tayfun Akin* \\ Department of Electrical and Electronics Engineering, Middle East Technical University, Ankara, Turkey
}

Received 22 October 2003; received in revised form 16 April 2004; accepted 24 April 2004

Available online 15 June 2004

\begin{abstract}
This paper presents a symmetrical and decoupled surface micromachined gyroscope fabricated by electroforming thick nickel on a glass substrate. The symmetric structure allows matched resonant frequencies for the drive and sense vibration modes for improved sensitivity, while the decoupled drive and sense oscillation modes prevents unstable operation due to mechanical coupling, resulting in a low zero-rate output drift. The use of a glass substrate instead of a silicon substrate reduces noise due to the parasitic signal coupling by two orders of magnitude, according to both simulation results in CoventorWare and measured results on fabricated devices. A capacitive interface circuit which is fabricated in a $0.8 \mu \mathrm{m}$ CMOS process is hybrid connected to the gyroscope, where the circuit has an input capacitance lower than $50 \mathrm{fF}$ and a sensitivity of $33 \mathrm{mV} / \mathrm{fF}$, which are currently limited by the parasitic capacitances due to hybrid wirebonding. It has been identified that the amount of parasitic capacitances must be lower than or at least in the same order with the electrical equivalent capacitance of the gyroscope for clear mechanical resonance characteristics to be obtained. Fabricated gyroscopes have close resonant frequencies for the drive and sense modes, as 37.2 and $38.3 \mathrm{kHz}$, respectively. Calculations on measured resonance values suggest that the fabricated gyroscope with $16 \mu \mathrm{m}$-thick structural layer provides a Brownian noise floor of $7.3^{\circ} / \mathrm{h} / \mathrm{Hz}^{1 / 2}$ at vacuum. Currently, the overall rate sensitivity of the gyroscope is limited to $96^{\circ} / \mathrm{h}$ in $50 \mathrm{~Hz}$ bandwidth for matched-frequency operation, and it can be decreased down to $56^{\circ} / \mathrm{h}$ in $50 \mathrm{~Hz}$ bandwidth, by improving the quality of the electrodeposited nickel.
\end{abstract}

(C) 2004 Elsevier B.V. All rights reserved.

Keywords: Symmetric gyroscope; Decoupled gyroscope; MEMS gyroscope; Capacitive interface circuit; Nickel electroforming

\section{Introduction}

Tactical-grade rate sensing applications require noise floor better than $0.01^{\circ} \mathrm{s}$ and bias drift as low as $1^{\circ} / \mathrm{h}$. These challenging requirements are difficult to achieve for MEMSbased Coriolis vibratory gyroscopes with current microfabrication technologies, unless dedicated mechanical designs and improved interface circuits are employed. In MEMS gyroscopes, it is essential to use matched resonance frequencies for the drive and sense mode vibrations for improving the sensitivity by the mechanical quality factor of the sense mode, which can be as high as a few thousand at vacuum [1]. This can be satisfied by designing symmetric suspensions for the drive and sense modes, which is also important to keep temperature-dependent drift small [2]. The disadvantage of symmetric suspensions is that they usually yield undesired mechanical coupling between drive and sense

\footnotetext{
* Corresponding author. Tel.: +90-312-210-2369; fax: +90-312-210-1261.

E-mail address: tayfun-akin@metu.edu.tr (T. Akin).
}

modes, resulting in a high bias drift. Bias drift can be minimized if the gyroscope has decoupled vibration modes [3]; however, these kind of gyroscopes usually have unsymmetrical suspensions and are subject to temperature-dependent drift. Some approaches aim to achieve both symmetrical and decoupled gyroscope $[4,5]$. We have reported a symmetrical and decoupled gyroscopes, called SYMDEC, implemented using the standard three-layer polysilicon surface micromachining (MUMPS) process of Cronos [5]. However, the performance of this gyroscope is limited due to the $2 \mu \mathrm{m}$ thickness of the structural polysilicon layer, resulting in very small sensor capacitances less than $10 \mathrm{fF}$. In addition, the conductive silicon substrate in the MUMPS process results in a very high parasitic capacitance of $3 \mathrm{pF}$, which significantly degrades the signal-to-noise ratio of the fabricated gyroscopes. This paper reports the development of a symmetric and decoupled gyroscope using nickel electroforming process on an insulating substrate [6,7]. The use of the insulating substrate decreases the parasitic capacitance by two orders of magnitude, while the nickel electroforming process allows to easily achieve structural layer thicknesses 
of around $16 \mu \mathrm{m}$ with a standard thick positive photoresist mold. In addition to the gyroscope, a new CMOS capacitive interface circuit is developed with low input capacitance and high sensitivity, and it is hybrid connected to the fabricated gyroscope to determine its performance.

\section{Analysis of symmetric and decoupled gyroscopes}

Fig. 1 illustrates the advantage of symmetric gyroscopes, where the drive $\left(w_{\mathrm{d}}\right)$ and sense $\left(w_{\mathrm{s}}\right)$ resonant frequencies are matched, considering that the frequency of the Coriolis force acting on the sense mode is at drive mode resonance frequency. When the two frequencies are separated from each other, the resultant Coriolis force acting along the sense mode yields a small displacement for that mode. On the other hand, when the two frequencies are closely matched, the sense mode motion is theoretically amplified by the mechanical quality factor of the sense mode.

The advantage of the matched drive and sense resonant frequencies can be verified using theoretical analysis. The general expression for the sense mode vibration amplitude
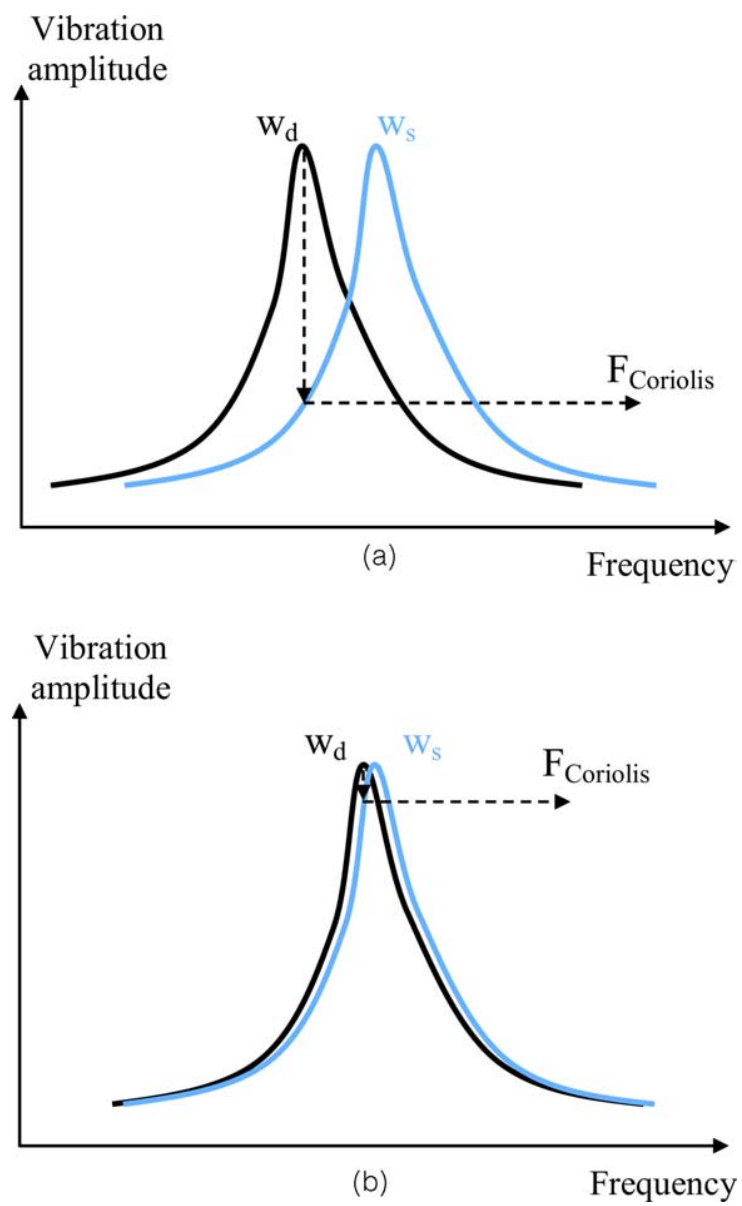

Fig. 1. Illustration showing the advantage of symmetric gyroscopes, where the drive $\left(w_{\mathrm{d}}\right)$ and sense $\left(w_{\mathrm{s}}\right)$ resonant frequencies are matched, considering that the frequency of the Coriolis force acting on the sense mode is at drive mode resonance frequency: the drive and sense mode resonance frequencies are (a) separated and (b) matched. of a Coriolis vibratory gyroscope is given by [8]

$$
\begin{aligned}
& Y\left(w_{\mathrm{d}}+w_{\mathrm{a}}\right)+Y\left(w_{\mathrm{d}}-w_{\mathrm{a}}\right) \\
& =\frac{2 \pi \Omega_{0} x_{0}\left(w_{\mathrm{d}}+w_{\mathrm{a}} / 2\right)}{\left(w_{y}^{2}-\left(w_{\mathrm{d}}+w_{\mathrm{a}}\right)^{2}\right)+j\left(w_{\mathrm{d}}+w_{\mathrm{a}}\right)\left(w_{y} / Q_{y}\right)} \\
& \quad+\frac{2 \pi \Omega_{0} x_{0}\left(w_{\mathrm{d}}-w_{a} / 2\right)}{\left(w_{y}^{2}-\left(w_{\mathrm{d}}-w_{\mathrm{a}}\right)^{2}\right)+j\left(w_{\mathrm{d}}-w_{\mathrm{a}}\right)\left(w_{y} / Q_{y}\right)}
\end{aligned}
$$

where $Y\left(w_{\mathrm{d}}+w_{\mathrm{a}}\right)$ and $Y\left(w_{\mathrm{d}}-w_{\mathrm{a}}\right)$ are the two phasor components [9] of the sense mode motion; $\Omega_{0}$ the applied angular rate input; $x_{0}$ the drive mode vibration amplitude; $Q_{y}$ the sense mode mechanical quality factor; $w_{\mathrm{d}}$ and $w_{y}$ are the drive and sense mode resonance frequencies, respectively; and $w_{\mathrm{a}}$ is the frequency of the time varying input angular rate. If the resonant frequencies of the drive and sense modes are accurately matched (that is, $w_{y} \cong w_{x} \cong w_{\mathrm{d}}$ ) and if the applied angular rate is constant $\left(w_{\mathrm{a}}=0\right)$, then the above equation simplifies to [10]

$Y\left(w_{\mathrm{d}}+w_{\mathrm{a}}\right)+Y\left(w_{\mathrm{d}}-w_{\mathrm{a}}\right) \approx \frac{4 \pi \Omega_{0} x_{0} Q_{y}}{j w_{\mathrm{d}}}$

showing that the sensitivity is amplified by the mechanical quality factor of the sense mode, $Q_{y}$. However, this sensitivity is valid only for constant angular rates. For time-varying angular rates, i.e., when $w_{\mathrm{a}}$ is not 0 , accurately matched resonant frequencies may not be desired. In these cases, Eq. (2) would have additional terms in its denominator, degrading the sensitivity with considerable amounts at high $w_{\text {a }}$ values. Also, the scale factors for the two sideband-displacements would be slightly different from each other. The phase of the sense mode displacement lags the drive-mode excitation force by $90^{\circ}$, and hence, it is in-phase with the drive-mode motion. Although the sensitivity is multiplied by the quality factor, the sense mode displacement magnitude is also very sensitive to changes in the bandwidth of the applied angular rate $\left(w_{\mathrm{a}}\right)$, limiting the angular signal bandwidth of the gyroscope. If there is a mismatch between the drive and sense mode resonant frequencies $\left(w_{\mathrm{d}}-w_{y} \gg w_{\mathrm{a}}\right.$ and $w_{\mathrm{d}}-w_{y} \ll$ $w_{\mathrm{d}}$ or $w_{y}$ ), then Eq. (1) reduces to

$Y\left(w_{\mathrm{d}}+w_{\mathrm{a}}\right)+Y\left(w_{\mathrm{d}}-w_{\mathrm{a}}\right) \approx \frac{2 \pi \Omega_{0} x_{0}}{\left(w_{y}-w_{\mathrm{d}}\right)}$

which states a sensitivity much less than that of Eq. (2). Here, the sense mode displacement is in-phase with the drive mode excitation force, and it is $90^{\circ}$ out-of-phase with the drive-mode displacement (excitation force and the sense mode motion leads the drive mode displacement by $90^{\circ}$ ). Although the sense mode displacement is not affected much from the changes in the angular signal frequency, $w_{\mathrm{a}}$, the sensitivity is reduced in this case. This analysis shows that it is essential to use matched drive and sense mode resonant frequencies for high sensitivity applications.

The major disadvantage of matched drive and sense mode resonant frequencies is the undesired mechanical coupling between the modes, resulting in a high bias drift, which can be eliminated with suitable decoupling mechanisms. Fig. 2 


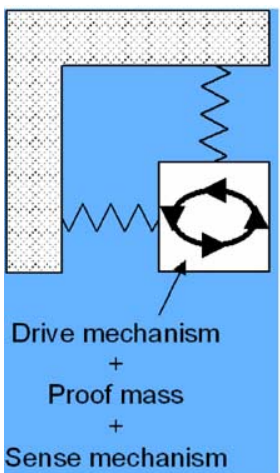

(a)

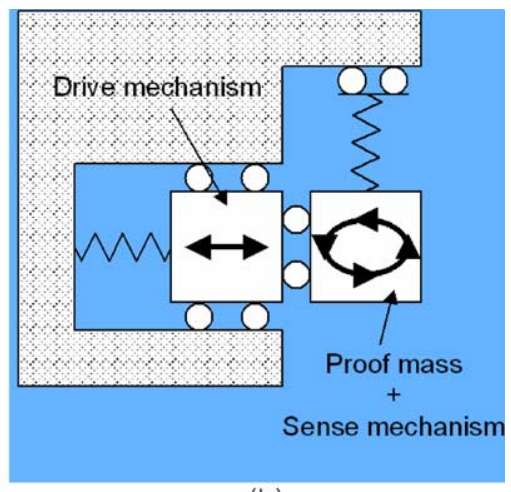

(b)

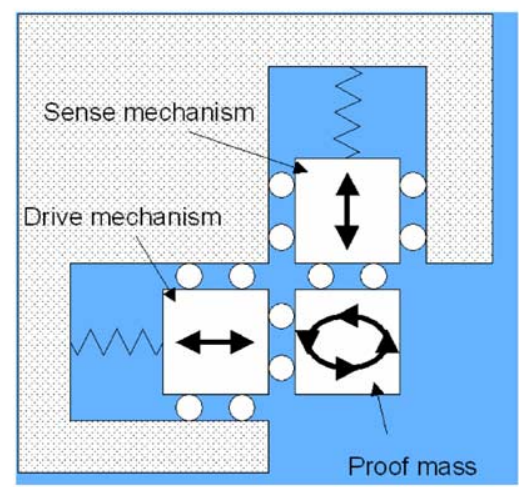

(c)

Fig. 2. Various decoupling mechanisms to achieve mechanical decoupling. (a) In the "no decoupling" case there is a continuous bias coupled to the sense mode and the drive-mode linearity is disturbed. (b) Decoupled-drive-mode removes the non-linearity but not the sense mode bias. The configuration in (c) completely solves both non-linearity and unwanted sense-mode bias.

presents various decoupling mechanisms to achieve mechanical decoupling. Fig. 2(a) shows the case where decoupling is not used, i.e., the drive and sense mechanisms and the proof mass all shares the same mass element and have two degree of freedom ( 2 d.f.) motion capability. However, in this case the linearity of the drive mode motion is disturbed when the sense mode also starts vibrating as a result of an applied angular rate input. Fig. 2(b) shows the case where decoupling is used only for the drive mode, i.e., the drive mode motion remains unaffected from sense mode vibrations since it is restricted to have only one degree of freedom (1 d.f.) motion along a predefined direction. However, any asymmetry in the device vibration patterns due to fabrication causes the drive mode vibrations directly transferred to the sense mode even in the absence of any angular rate input, resulting in a certain zero-input bias. Fig. 2(c) provides the effective solution to this problem, where the drive and sense mechanisms and the proof mass are all discrete mechanical elements, attached to each other via elastic flexures; only the proof mass has 2 d.f. motion with the drive and sense mode motions are restricted to 1 d.f. This way, the drive and sense mode vibrations does not directly affect each other, resulting in linear drive mode vibrations as well as a low zero-input bias.

This analysis shows that it is important to have a symmetric gyroscope structure for high sensitivity, while the sense and drive modes of the gyroscope should be decoupled for low bias drift. Section 3 explains the structure of the symmetric and decoupled gyroscope developed in this study.

\section{Symmetric and decoupled gyroscope structure}

Fig. 3 shows the perspective view of the symmetric and decoupled (SYMDEC) gyroscope structure. The drive mode ( $x$-axis) of the gyroscope is electrostatically excited to oscillate at its resonance frequency. When an angular rate input is applied about the $z$-axis, the sense mode also starts vibrating due to the Coriolis coupling from the drive mode, and this vibration is sensed with a capacitive interface circuit. This structure has both symmetric suspensions and mechanically decoupled oscillation modes. The anchors of the structure are placed at the outermost corners and connected to the movable drive and sense electrodes with the help of suspension beams in such a way that the drive electrodes cannot vibrate along the oscillation direction of the sense electrodes and vice versa, therefore, the mechanical decoupling is achieved. In addition, the suspension beams support-

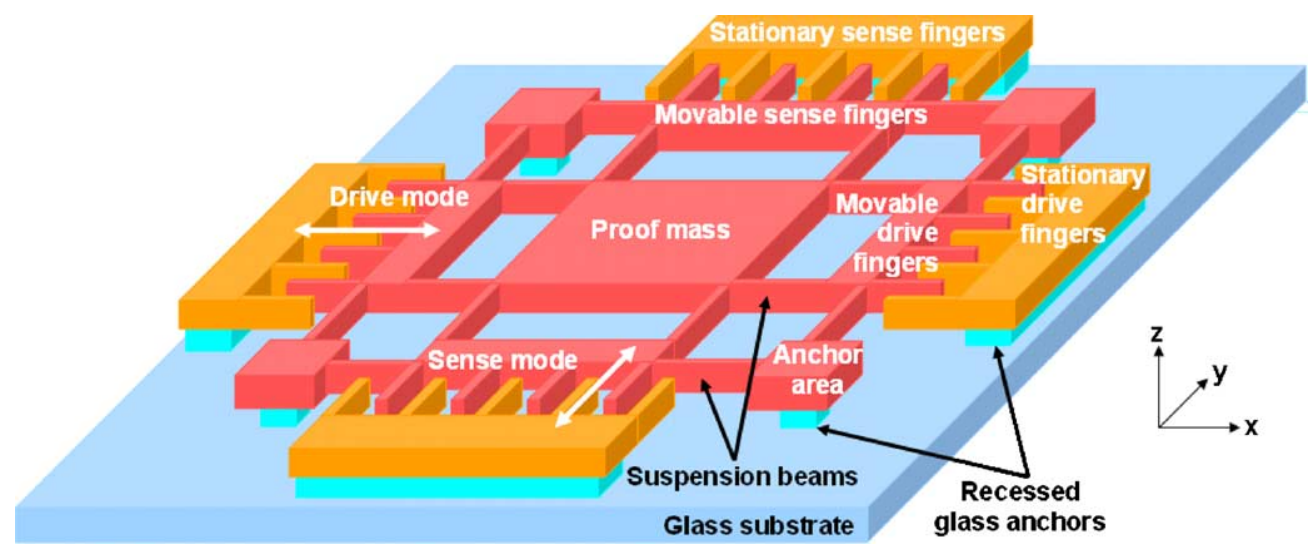

Fig. 3. The perspective view of the symmetric and decoupled (SYMDEC) gyroscope structure. 
ing the proof mass are not attached to the anchors directly, rather the proof mass is supported by the beams attached to the movable drive and sense electrodes. This way, symmetry of the structure is preserved, while the drive and sense electrode motions are kept independent from each other.

\section{Simulation results}

The operation and performance of the symmetric and decoupled gyroscope are verified with simulations using lumped element models in SPICE and finite element models in CoventorWare. First, lumped element models are developed in SPICE to analyze the effect of parasitic capacitances when conducting and insulating substrates are used. Second, finite element models are constructed in CoventorWare to analyze the mode shapes and verify decoupled operation. Fig. 4 shows the simulations performed in SPICE on the lumped element model of the gyroscope. These simulations show that the signal-to-noise ratio of any capacitive resonator improves
Table 1

Chemical composition and operating conditions of the low-stress nickel electroplating solution

\begin{tabular}{ll}
\hline Chemical/Plating Parameter & Amount/Value \\
\hline Nickel sulfamate $\left(\mathrm{NiSO}_{3} \mathrm{NH}_{2} \cdot 4 \mathrm{H}_{2} \mathrm{O}\right)$ & $500 \mathrm{~g} / \mathrm{L}$ \\
Boric acid $\left(\mathrm{H}_{3} \mathrm{BO}_{3}\right)$ & $30 \mathrm{~g} / \mathrm{L}$ \\
Nickel chloride $\left(\mathrm{NiCl}_{2}\right)$ & $2 \mathrm{~g} / \mathrm{L}$ \\
Plating parameter & Value \\
Plating temperature & $50^{\circ} \mathrm{C}$ \\
Current density & $5 \mathrm{~A} / \mathrm{dm}^{2}$ \\
\hline
\end{tabular}

Table 2

Summary of device mechanical dimensions and electrical properties

\begin{tabular}{ll}
\hline Proof mass size & $1.9 \mathrm{~mm} \times 1.9 \mathrm{~mm}$ \\
Structural layer thickness & $16 \mu \mathrm{m}$ \\
Offset over substrate & $2 \mu \mathrm{m}$ \\
Aspect ratio & $5.3: 1$ \\
Drive/sense electrode capacitances & $65-100 \mathrm{fF}^{\mathrm{a}}$ \\
Parasitic capacitances & $<30 \mathrm{fF}$ \\
\hline
\end{tabular}

${ }^{\mathrm{a}}$ For different prototypes.
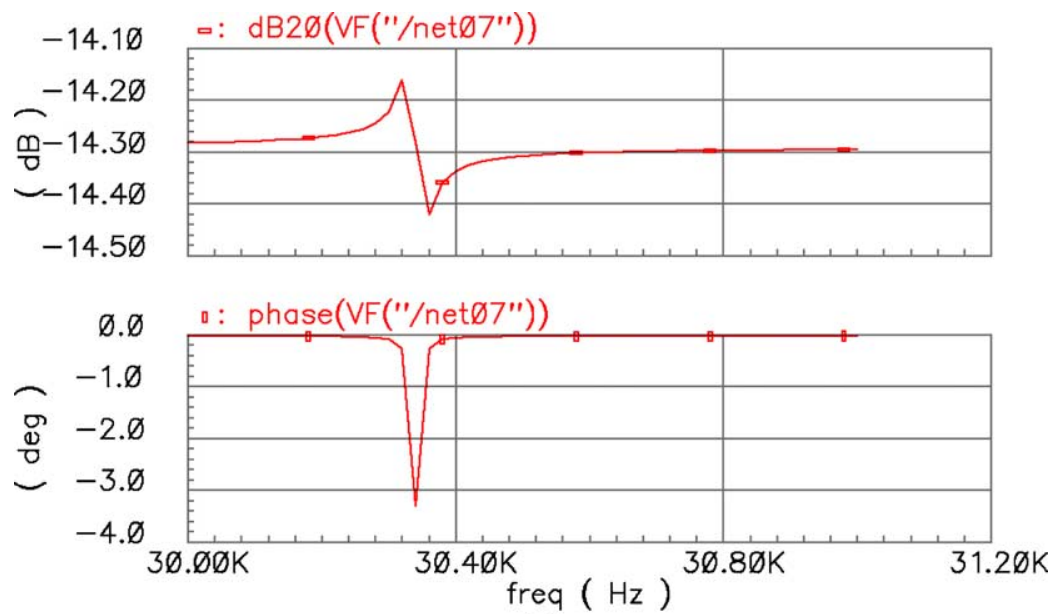

(a)
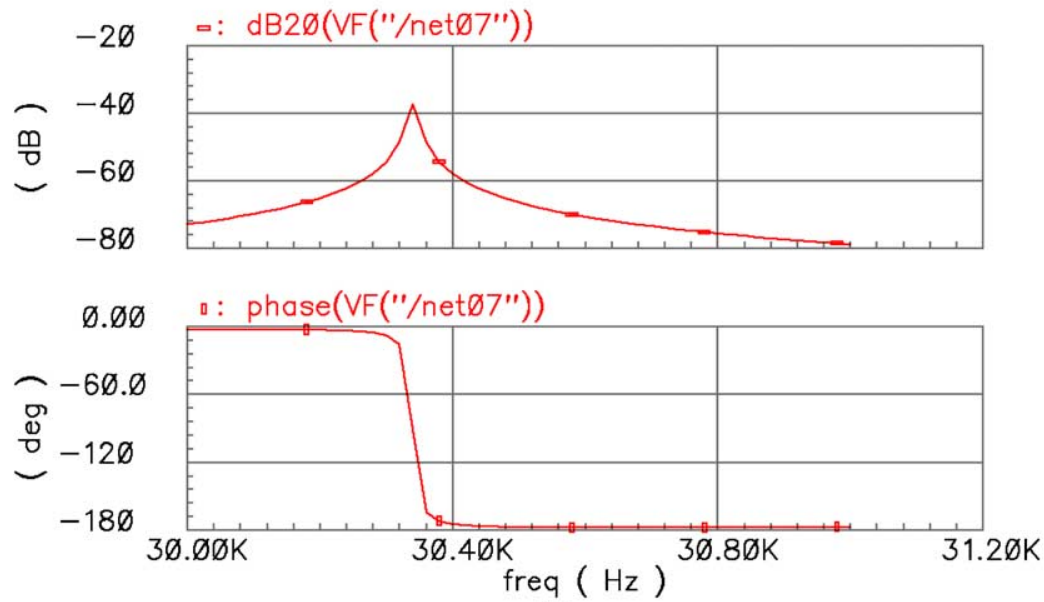

(b)

Fig. 4. The simulations performed by SPICE on the lumped element model of the gyroscope, showing the effect of parasitic capacitances when (a) conducting and (b) insulating substrates are used. 


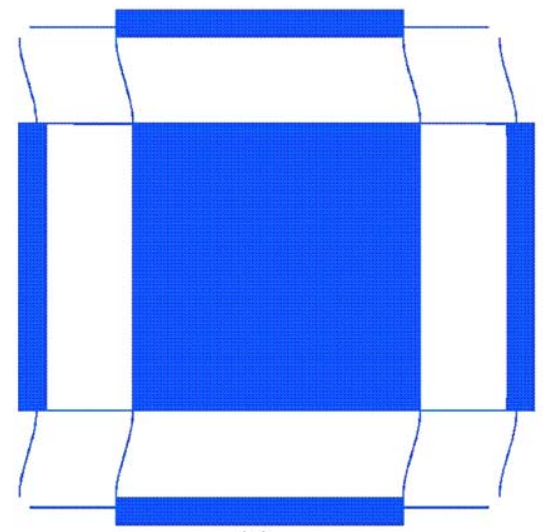

(a)

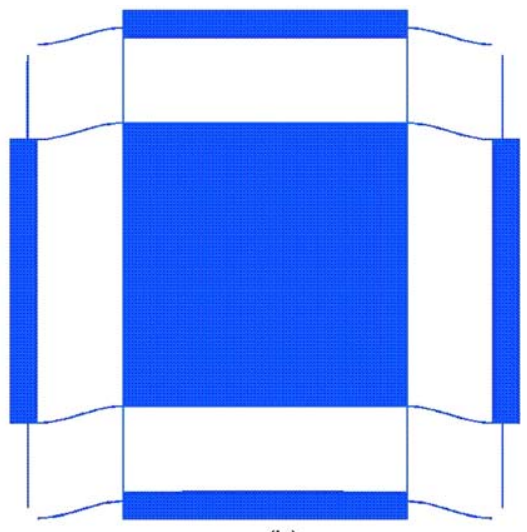

(b)

Fig. 5. CoventorWare FEM simulations, where the (a) drive and (b) sense mode resonance frequencies are matched at about $37 \mathrm{kHz}$.

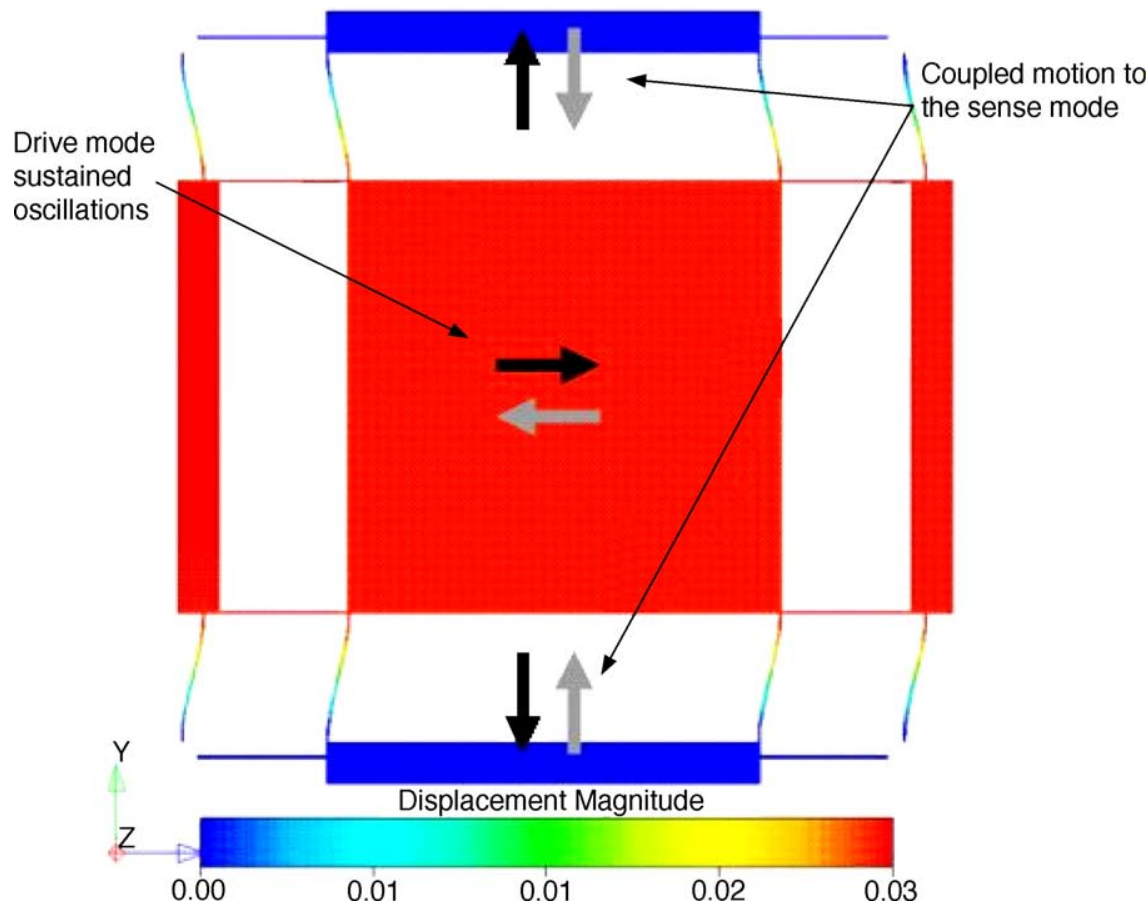

Fig. 6. The FEM simulation result showing that the mechanical coupling from the drive mode to the sense mode is less than $2 \%$ of the drive mode vibration amplitude.

by two orders of magnitude with the use of insulating (glass) substrate instead of a conductive substrate. Fig. 5 shows CoventorWare FEM simulations, where the drive and sense mode resonance frequencies of the two modes are matched at about $37 \mathrm{kHz}$. These simulations take into account the effects of etch-holes, comb-fingers, and residual stress of the structural nickel layer, and therefore provide results very similar to the measured resonance frequencies. Fig. 6 presents the FEM simulation result showing that the mechanical coupling from the drive mode to the sense mode is less than $2 \%$ of the drive mode vibration amplitude. Since this small coupling affects the both sides of the sense electrodes similarly, this coupling is suppressed using a differential readout scheme. It should be noted that the frequency of the coupling signal is twice that of the drive mode, since the sense mode electrode moves twice when the drive mode electrode moves once, due to the symmetric nature of the gyroscope structure. This allows further suppression of the coupling signal with the readout electronics, resulting in very low quadrature error signal. These simulations show that the structure proposed in this study provides both symmetrical and decoupled operation.

\section{Implementation and test results}

The symmetrical and decoupled gyroscope in this study is implemented using a simple electroforming process on 


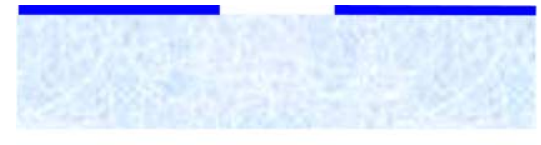

(a) Cr/Au metallization using lift-off over glass substrate

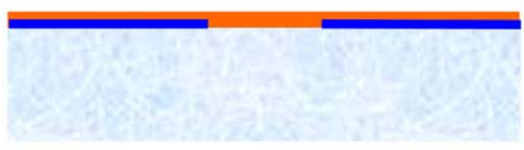

(b) Sputter coat 2000A-thick copper seed layer

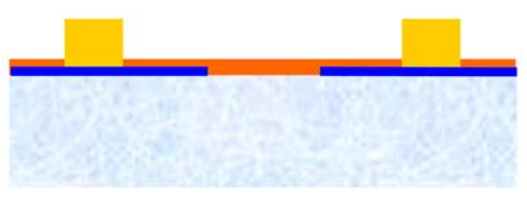

(c) Coat and pattern $5 \mu$ m-thick negative tone photoresist

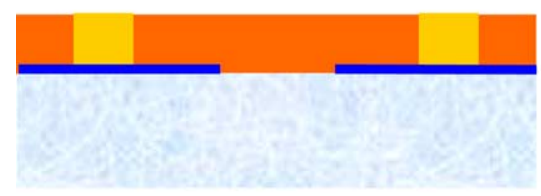

(d) Electroplate $2 \mu \mathrm{m}$ thick copper as the sacrificial layer

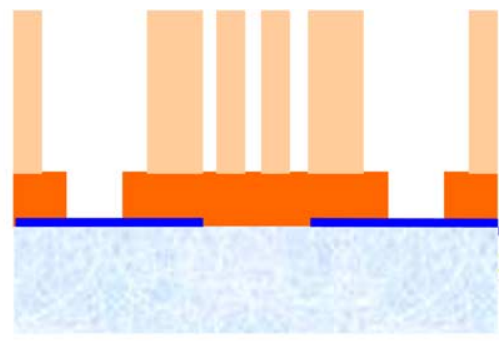

(e) Coat and pattern $18 \mu$ m-thick structural photoresist

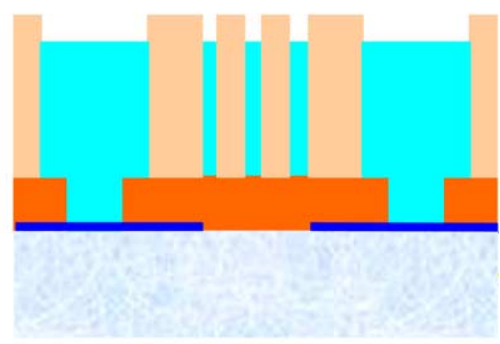

(f) Electroplate $16 \mu$ m-thick nickel structural layer

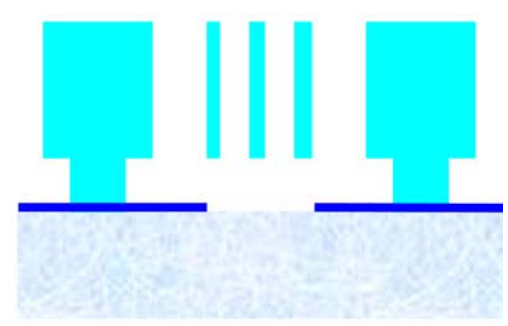

(g) Release the structures by removing structural mold and sacrificial copper.

Fig. 7. Fabrication steps of the gyroscopes with nickel electroforming process.

a glass substrate. Fig. 7 shows the fabrication steps, where only three masks are used. First, a Cr/Au interconnect metallization layer is deposited and patterned over the Pyrex glass substrate by the lift-off technique. Next, a $2000 \AA$ A-thick copper seed layer is sputter-coated on the whole wafer surface and patterned by wet etching in the anchor regions where structural nickel is to be electroplated. Removal of copper from the anchor regions prevents any excessive undercut during the selective etching of the sacrificial copper in the final release step. Then, a negative-tone photoresist is coated and patterned in the anchor regions with sufficient side-wall angle to prepare a mold for electrodeposition of $2 \mu \mathrm{m}$-thick copper sacrificial layer. Following that, an $18 \mu \mathrm{m}$-thick photoresist mold (SJR5740) is coated and patterned on top of the copper sacrificial layer. Into this mold, a $16 \mu \mathrm{m}$-thick nickel layer is electrodeposited using a low-stress nickel sulfamate bath with the chemical compositions shown in Table 1. Finally, the mold and copper sacrificial layers are removed to release the nickel microstructures suspended over the glass substrate.

Fig. 8 shows the SEM picture of one of the fabricated gyroscopes. The device measures approximately $2 \mathrm{~mm}$ on one side including its fixed electrodes, and the size of the proof mass is $1 \mathrm{~mm} \times 1 \mathrm{~mm}$. Fig. 9 shows the closer SEM pictures of the comb fingers of the gyroscope. The thickness of the structural layer is currently $16 \mu \mathrm{m}$, limited by the thickness of the photoresist mold. Currently, it is possible to obtain $1 \mu \mathrm{m}$ gap spacing and $3 \mu \mathrm{m}$ feature size for the fabricated devices, providing an aspect ratio greater than 5 and electrode capacitances in the order of 80-100 fF. Table 2 provides a summary of device mechanical dimensions and some electrical properties.

The capacitance from the drive mode to the sense mode is measured as $87 \mathrm{fF}$ by a precision impedance analyzer (HP4294A). When the expected capacitance of the comb fingers, which is around $65 \mathrm{fF}$ is extracted, the parasitic ca- 


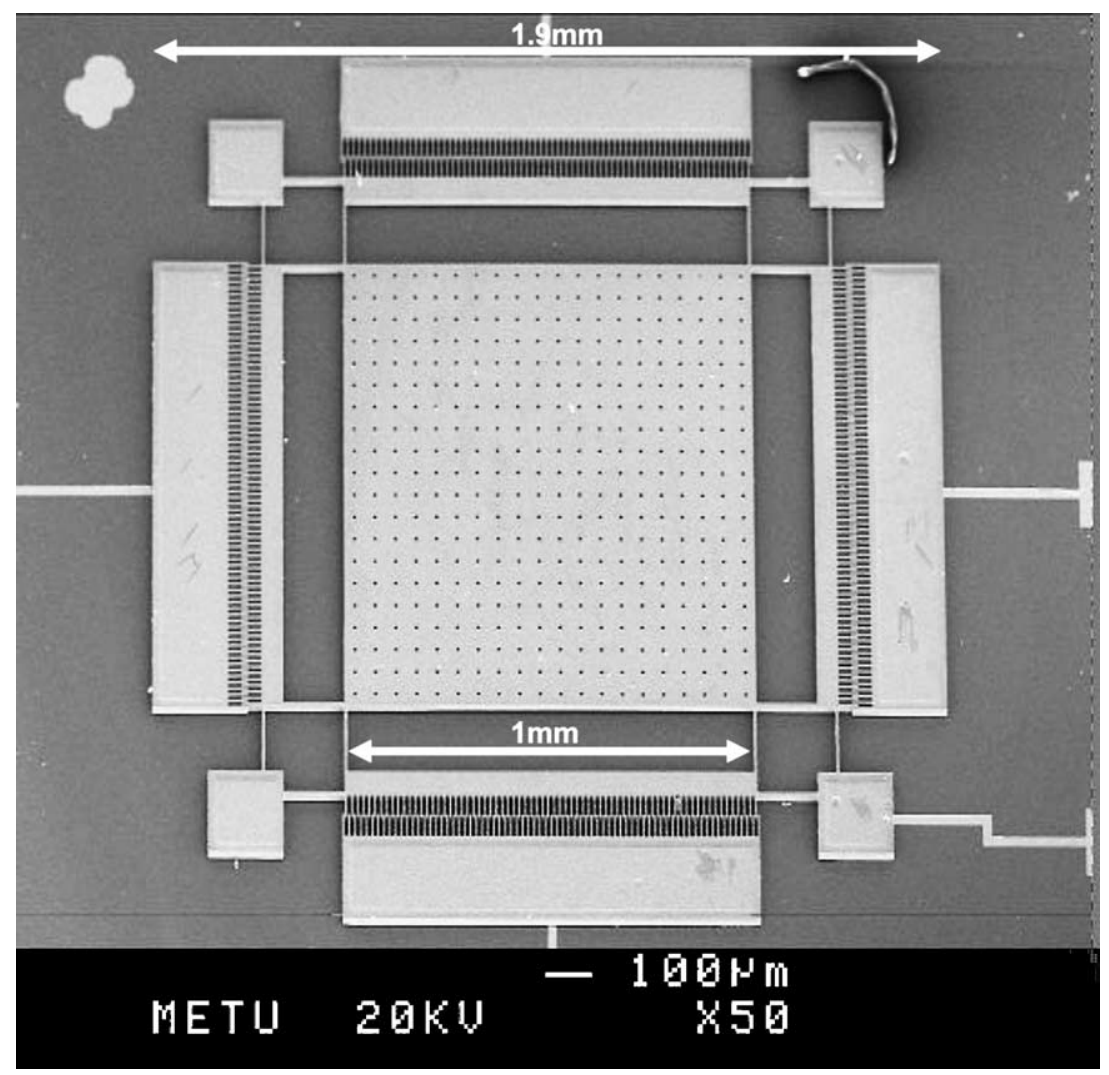

Fig. 8. SEM picture of one of the fabricated gyroscopes. The device measures approximately 2 mm on one side including its fixed electrodes, and the size of the proof mass is $1 \mathrm{~mm} \times 1 \mathrm{~mm}$.

pacitance is determined as only $22 \mathrm{fF}$, i.e., very small due to the insulating glass substrate, greatly reducing the electrical cross-coupling through the substrate. Measurements show that, the parasitic coupling is mostly due to the hybrid wirebonding of the gyroscope and readout circuit chips.

The fabricated gyroscopes are attached to a capacitive interface circuit which is fabricated in a standard $0.8 \mu \mathrm{m}$ CMOS process. Fig. 10 shows the optical microscope photograph of the fabricated interface circuit. The circuit fits into a small area of $150 \mu \mathrm{m} \times 250 \mu \mathrm{m}$. Fig. 11 shows the schematic of the interface circuit. The circuit uses unity-gain buffer topology [11] to buffer the output of the gyroscope, which is a high-impedance-node since the sensor capacitances are in the order of few tens of femtofarads. The input impedance of the buffer-type capacitive interface circuit is maximized by minimizing the input capacitance of the interface [12]. There are various sources that contribute to the input capacitance of the interface circuit, including parasitic capacitances from the input pad and interconnect lines, the input capacitance of the biasing scheme, and the transistor capacitances of the input transistor.

The parasitic capacitances associated with the input pad and interconnect lines are cancelled by the bootstrapping method [11], where a metal shield is placed underneath the input pad and the interconnect lines, and this shield is driven by the output of the buffer stage. The sensor output voltage expression shown in Fig. 12 can be determined as

$V_{\text {out }}=5^{v} A_{\text {buffer }} \frac{2 \Delta C}{2 C+C_{\mathrm{P}}^{\prime}}$

where $A_{\text {buffer }}$ is the gain of the buffer circuit, $C$ the sense capacitance of the gyroscope, $\Delta C$ the change in the sense capacitance, and $C_{\mathrm{P}}^{\prime}$ is the new input capacitance appearing at the input of the buffer when bootstrapping is used, which is expressed as

$C_{\mathrm{P}}^{\prime}=C_{\mathrm{P}}\left(1-A_{\text {buffer }}\right)+C_{\mathrm{in}}$

where $C_{\mathrm{P}}$ is the parasitic capacitance at the input node and $C_{\text {in }}$ the capacitance of the buffer and its biasing circuit. The $C_{\mathrm{P}}$ is generally very large, in the order of picofarads, while $C$ is only tens of femtofarads. The effect of $C_{\mathrm{P}}$ is significantly reduced as it is multiplied by the $\left(1-A_{\text {buffer }}\right)$ term and $A_{\text {buffer }}$ is very close to unity. Therefore, $C_{\mathrm{P}}^{\prime}$ is mostly determined by $C_{\mathrm{in}}$, which can be made small with the proper design of the buffer circuit and its biasing network. In this case, the output voltage of the sensor in Eq. (4) can be made at least few orders of magnitude larger when bootstrapping is used to cancel the large parasitic capacitances at the input of the interface circuit.

The amount of output voltage is primarily determined by $C_{\text {in }}$, which can be reduced by minimizing the input capacitance of the buffer circuit and the capacitance associated 


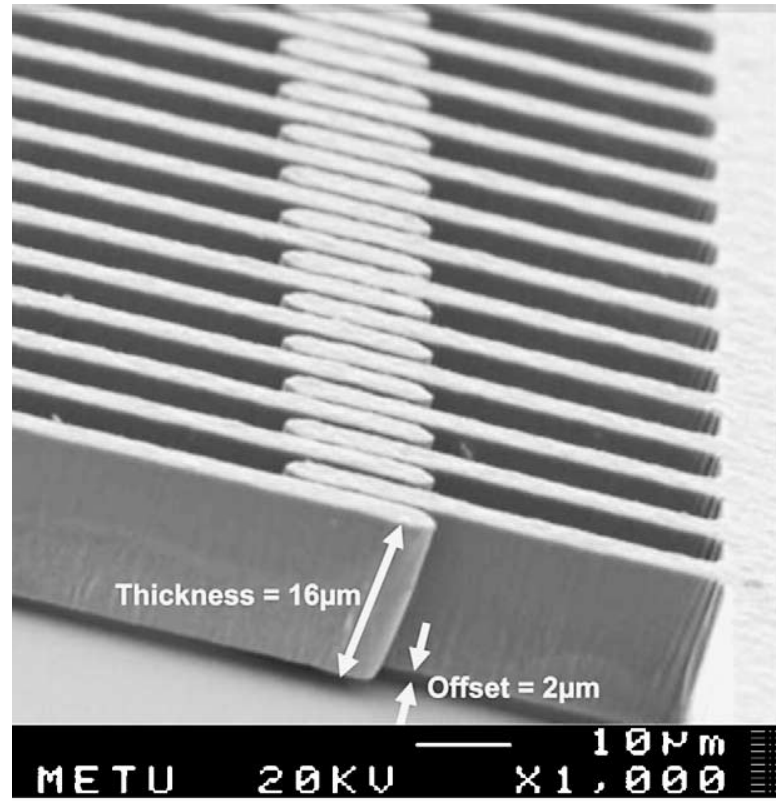

(a)

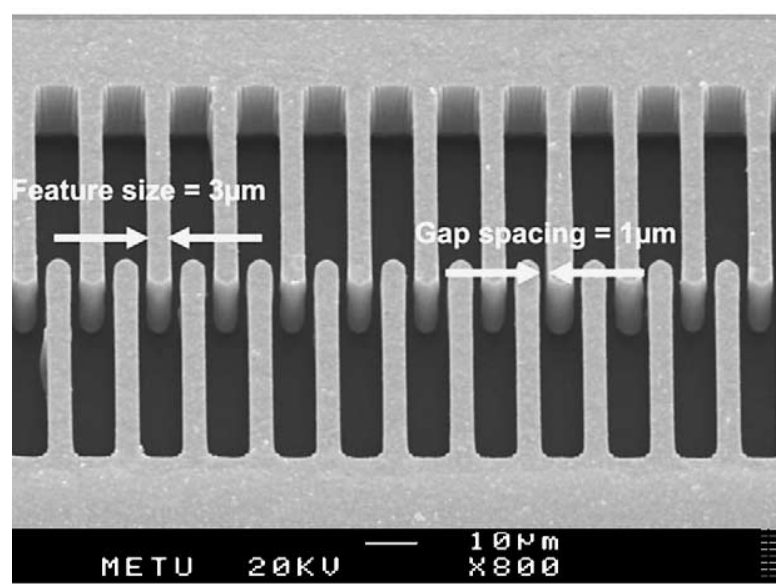

(b)

Fig. 9. Closer SEM pictures of the comb fingers of the gyroscope. (a) The thickness of the structural layer is currently $16 \mu \mathrm{m}$, limited by the thickness of the photoresist mold. (b) It is possible to obtain $1 \mu \mathrm{m}$ gap spacing and $3 \mu \mathrm{m}$ feature size for the fabricated gyroscopes.

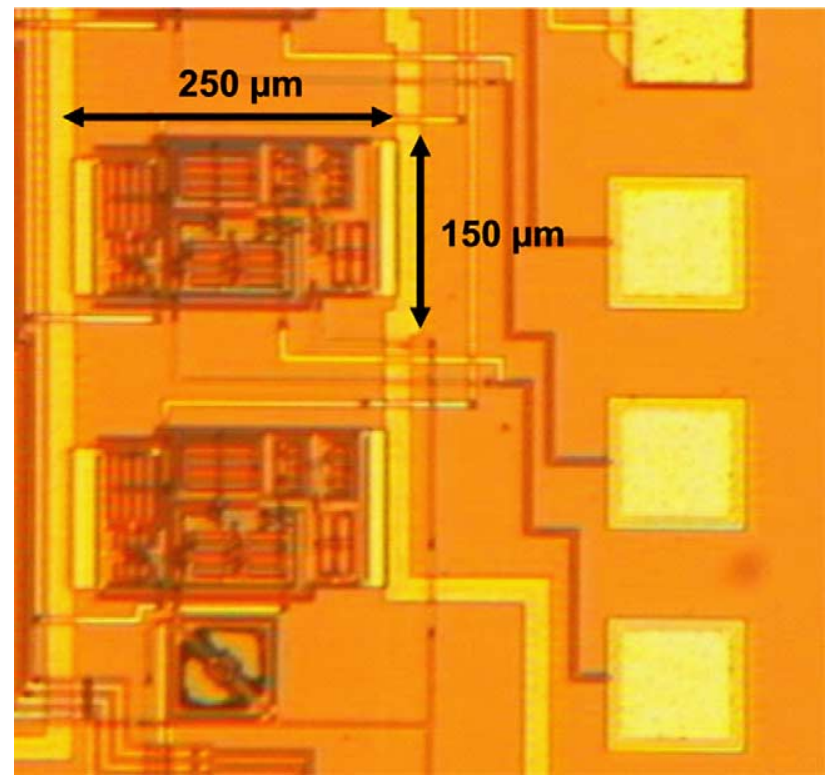

Fig. 10. Optical microscope photograph of the fabricated interface circuit.

with the biasing circuit. The capacitance associated with the biasing circuit is minimized by using a minimum size PMOS transistor operating in the subthreshold region, which provides high-impedance for the input-node dc bias while keeping the transistor capacitances in the order of few femtofarads. The input capacitance of buffer circuit is minimized by mirroring the voltage at the source of the input transistor to its drain, which cancels the most dominant input capacitance, namely the gate-drain capacitance. The gate-source and gate-bulk capacitances are already smaller than a fraction of a femtofarad, and therefore, do not affect the input capacitance much due to the source-follower topology. These improved buffer topology results in a simulated input capacitance of less than $3 \mathrm{fF}$, sufficiently low input capacitance to effectively buffer the high-impedance gyroscope output. However, in practice, the input capacitance of the circuit has been measured as $50 \mathrm{fF}$, limited by the hybrid-wirebonding.

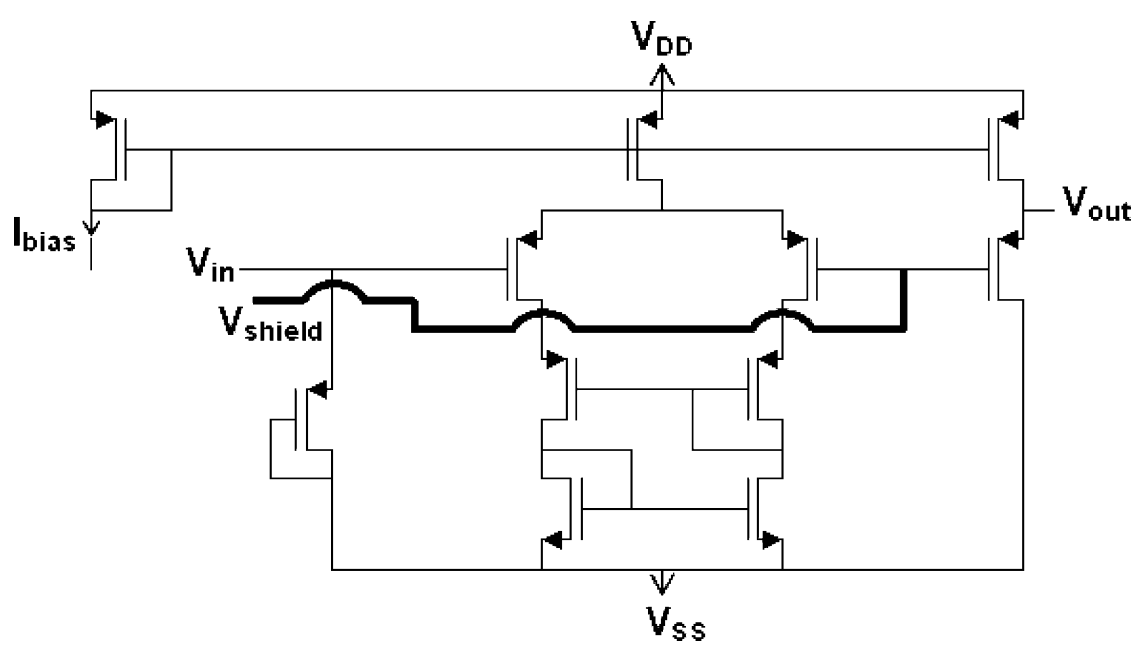

Fig. 11. Schematic view of the designed capacitive interface circuit. 


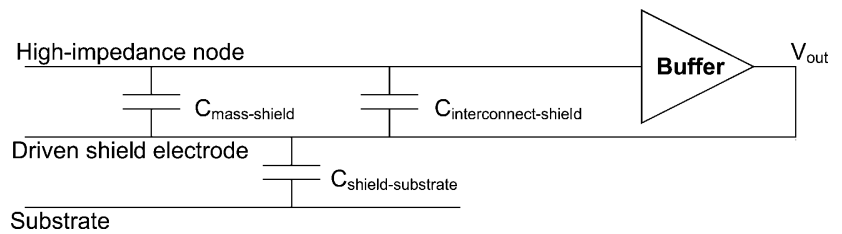

Fig. 12. Bootstrapping method for the cancellation of various parasitic capacitances at the input of the capacitive interface circuit. The output of the unity-gain buffer drives a shield underlying the high-impedance input node, effectively canceling parasitic capacitance from input node to substrate.

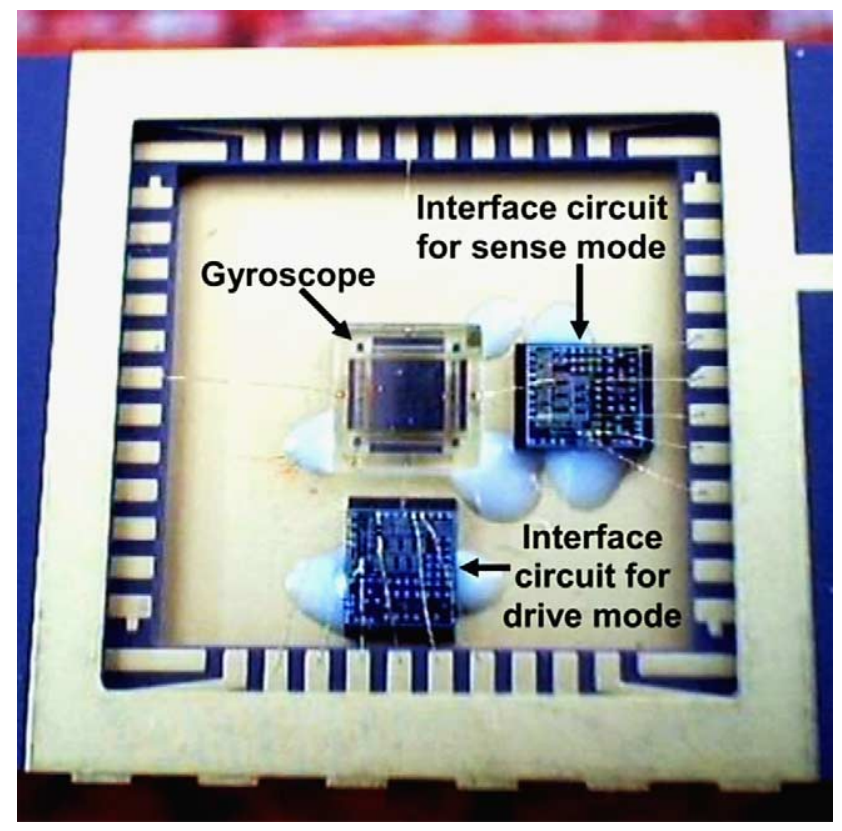

Fig. 13. Photograph of two CMOS interface chips wirebonded to a gyroscope chip.
Two of the CMOS capacitive interface circuits are attached to a fabricated gyroscope, as shown in Fig. 13, for testing the resonance characteristics of the drive and sense modes. Fig. 14 shows the input excitation signal for drive mode resonance of the gyroscope and corresponding output signal picked by the interface circuit. The peak-to-peak value of the drive mode excitation signal is $4.3 \mathrm{~V}$, while the peak-to-peak value of the measured signal at the drive mode output is $1.1 \mathrm{~V}$. At this condition, the vibration amplitude is measured as $4 \mu \mathrm{m}$, corresponding to a capacitance change of $32 \mathrm{fF}$ at the gyroscope output. By dividing the interface circuit output signal by this capacitance change, the sensitivity of the fabricated interface circuit is determined as $33 \mathrm{mV} / \mathrm{fF}$.

Fig. 15 shows the measured resonance frequencies of the drive and sense modes of the gyroscope as 37.2 and $38.3 \mathrm{kHz}$, respectively, by using the HP4395A network analyzer and a $40 \mathrm{~V}$ dc polarization voltage. The anti-resonance peaks in these measurements are due to parasitic capacitances associated with the hybrid wire-bonding of the gyro and CMOS chips. These anti-resonance peaks make it difficult to lock onto the mechanical resonances, when the gyroscope is inserted into a self-oscillation loop. Therefore, it is necessary to remove these anti-resonance peaks from actual mechanical resonance peaks, which is possible after determining the source of the anti-resonance peaks.

The major source for anti-resonance peaks is simply the stray capacitances through substrate or wirebonding, as verified by analyzing the lumped-element model of the fabricated gyroscope hybrid-connected to the capacitive interface circuit. Fig. 16 shows this lumped-element model, where, the $L_{x}, R_{x}$, and $C_{x}$ denote the equivalent inductance, resistance, and capacitance of the gyro lumped model, respec-

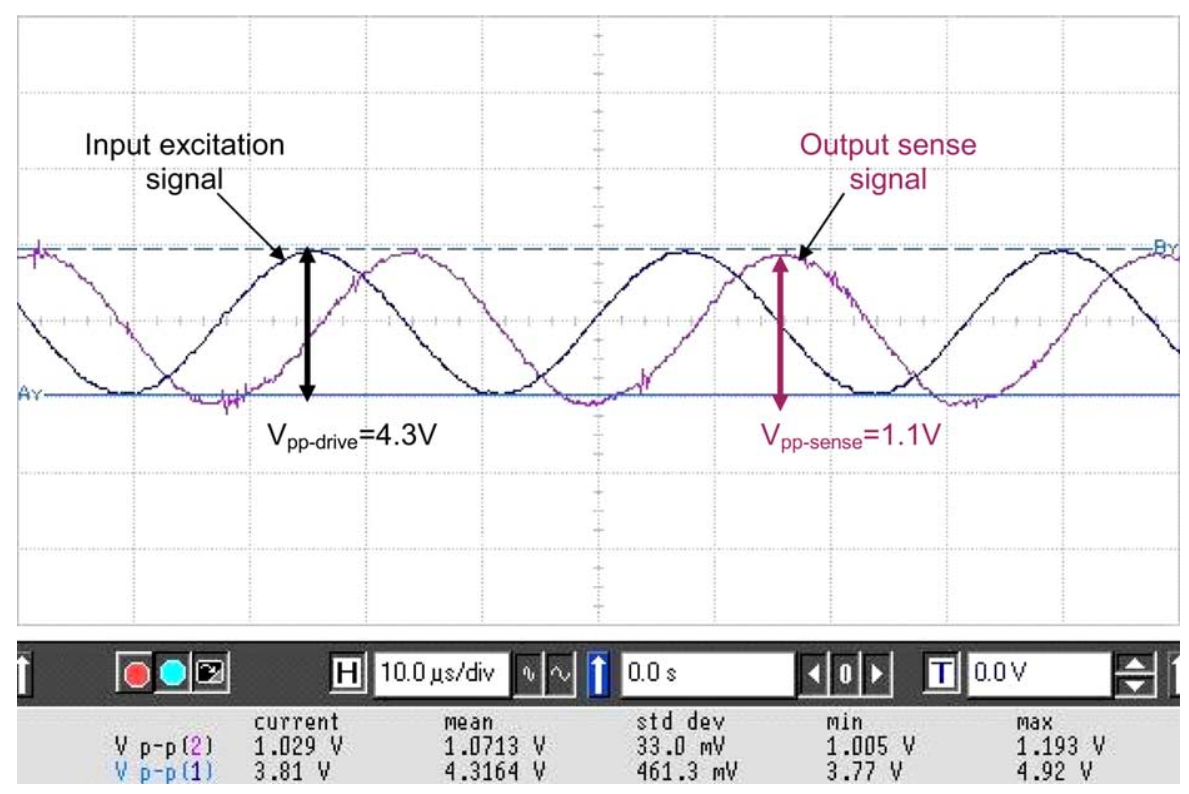

Fig. 14. The input excitation signal for drive mode resonance of the gyroscope and corresponding output signal picked by the interface circuit. 

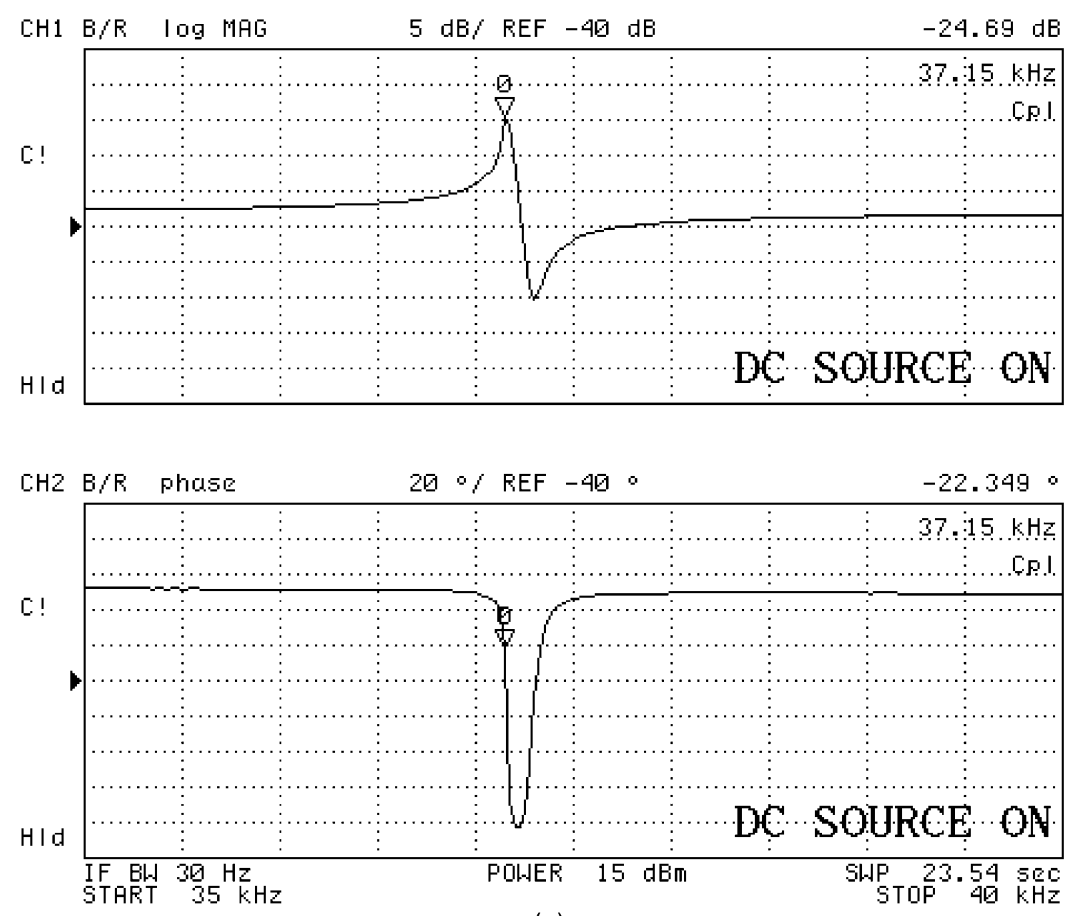

(a)
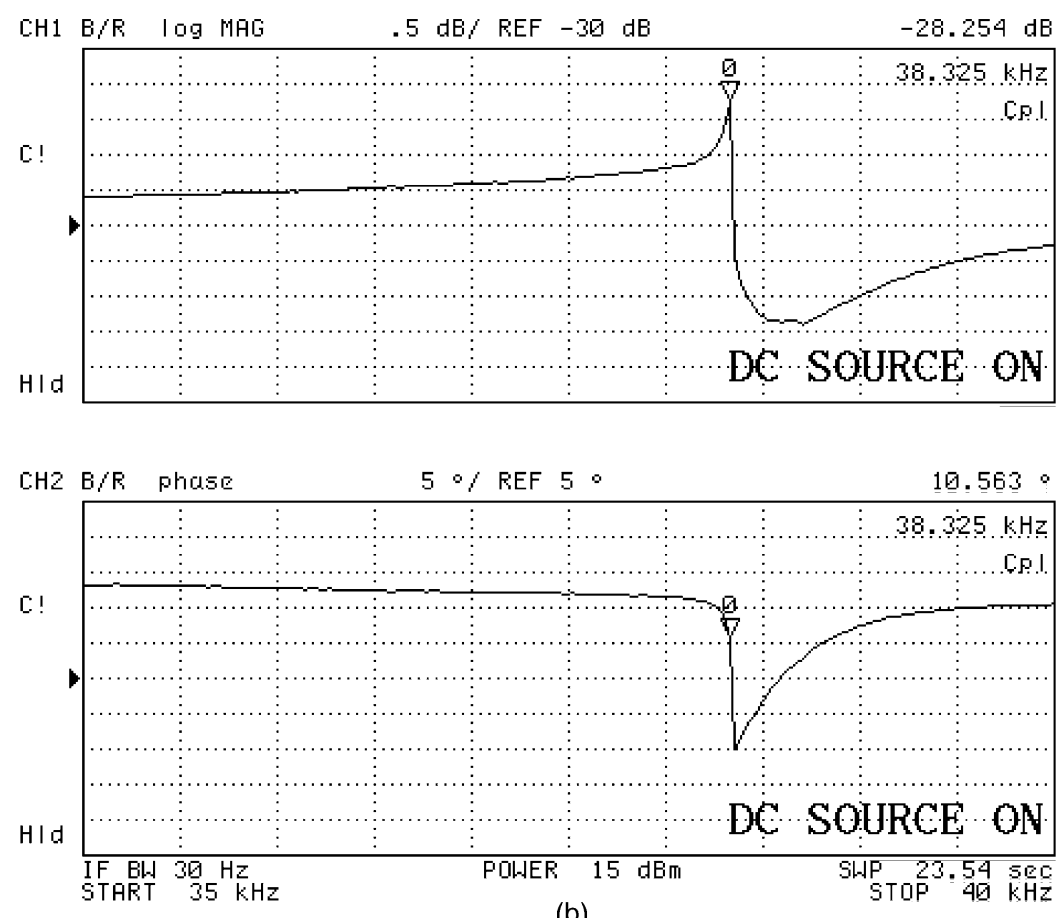

(b)

Fig. 15. The measured resonance frequencies of the drive and sense modes of the gyroscope as 37.2 and $38.3 \mathrm{kHz}$, respectively, by using the HP4395A network analyzer and a $40 \mathrm{~V}$ dc polarization voltage.

tively. These parameters can be obtained using the following relations:

$L_{x}=\frac{m}{n^{2}}, \quad R_{x}=\frac{b}{n^{2}}, \quad C_{x}=\frac{n^{2}}{k}$

where $m$ is the mass, $b$ the damping factor, $k$ the mechani- cal spring constant, and $n$ is the electromechanical conversion factor, which is a function of the $\partial C / \partial x$ parameter of the gyroscope and the dc polarization voltage applied to the proof mass. The $\partial C / \partial x$ parameter is the capacitance change per unit displacement of the movable electrode, and it is determined by the aspect ratio and geometry of the fabricated 


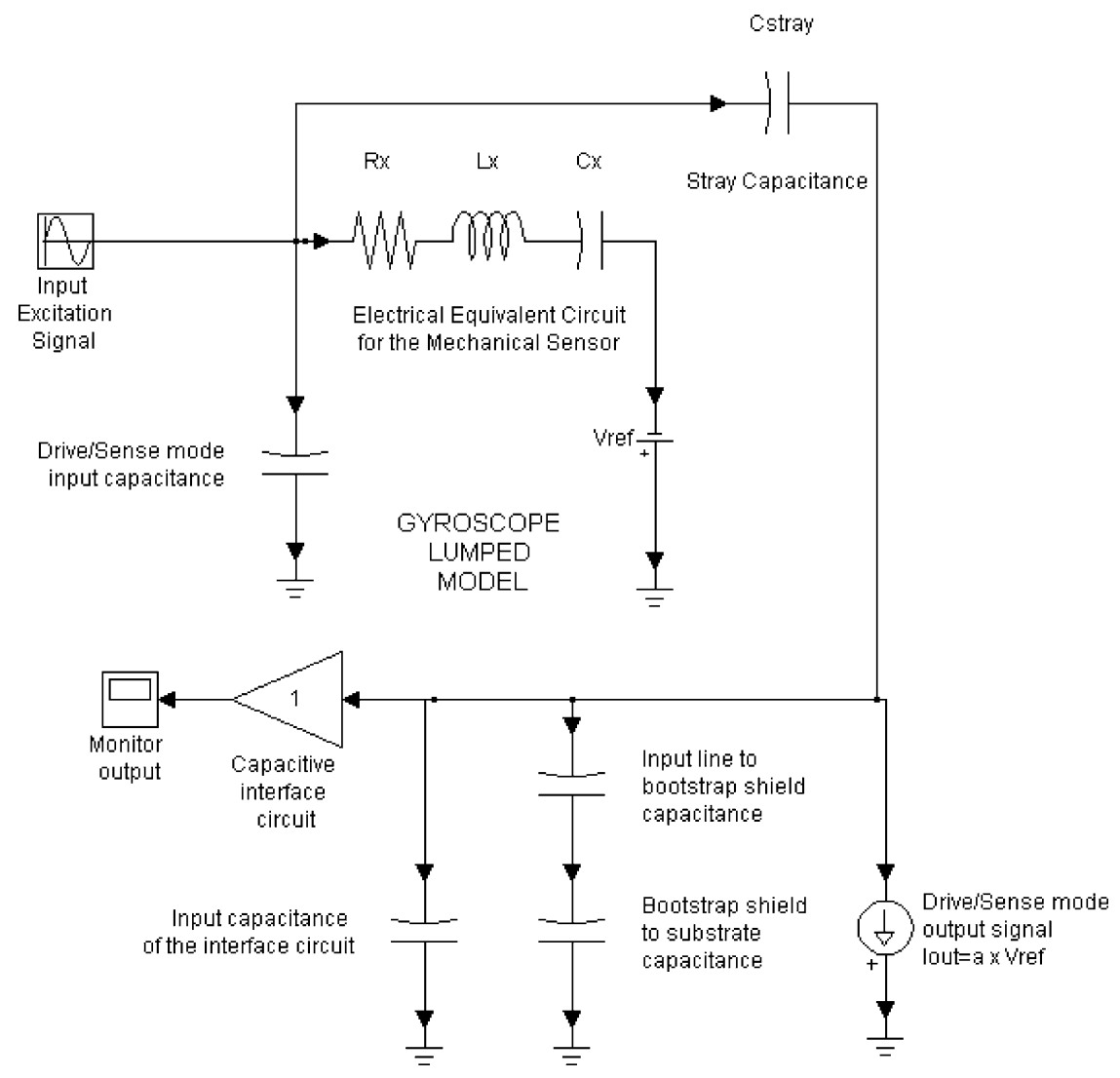

Fig. 16. The lumped-element model for the fabricated gyroscope hybrid-connected to the designed capacitive interface circuit.
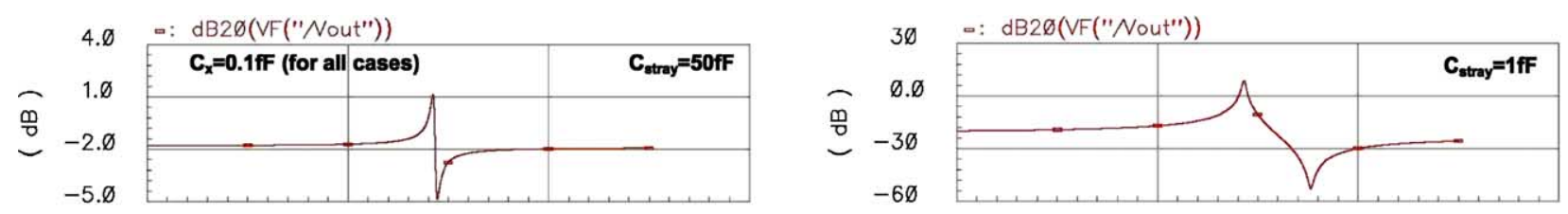

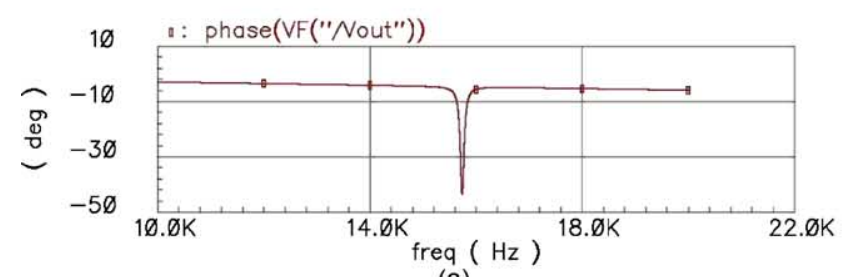

(a)

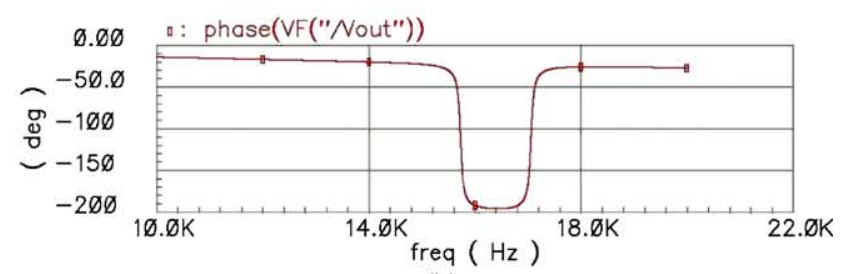

(b)
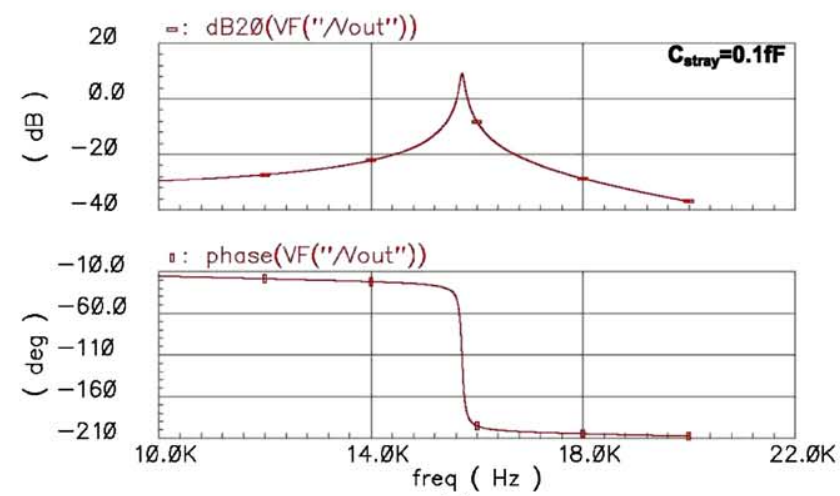

(c)

Fig. 17. The simulation results when the gyroscope equivalent capacitance in the lumped model is increased. 

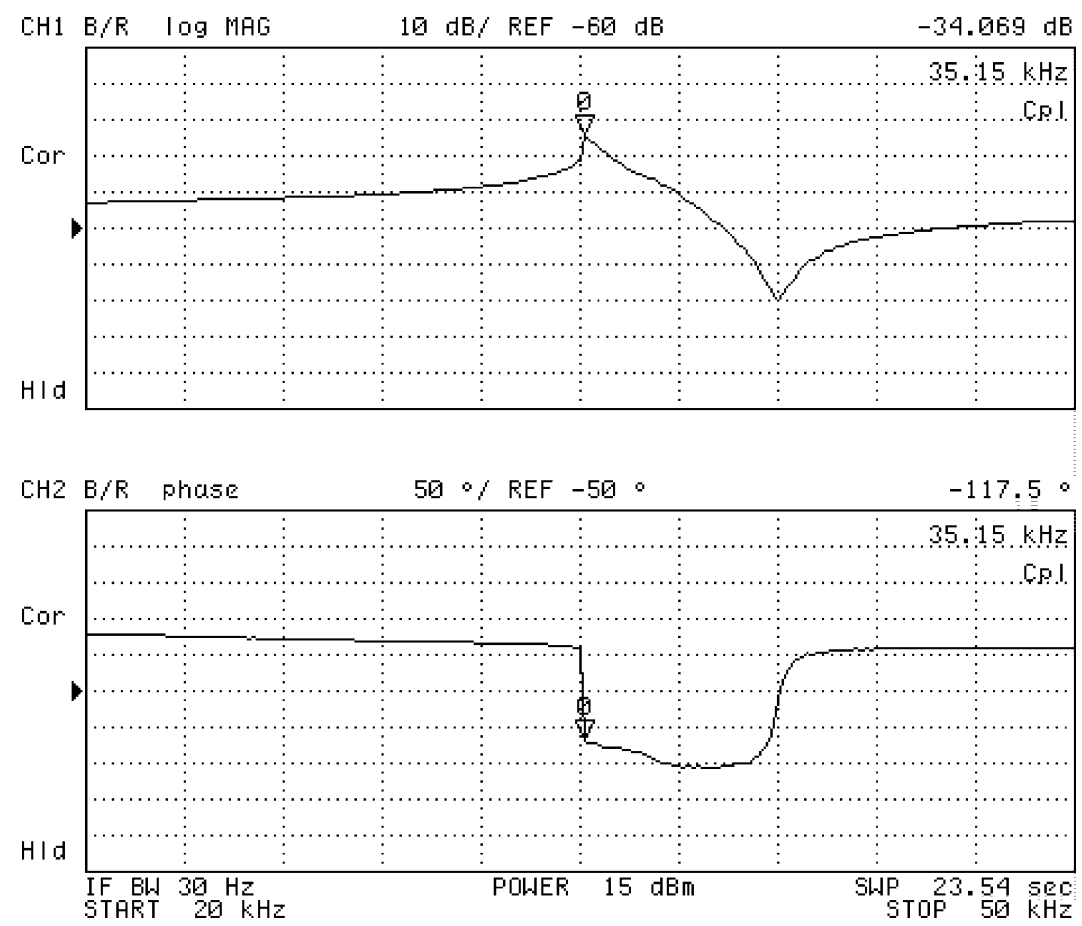

Fig. 18. Measured resonance characteristics of the gyroscope when the dc polarization voltage of the gyroscope is increased up to $100 \mathrm{~V}$.

structures. The dc polarization voltage, however, can be adjusted to tune the electromechanical conversion parameter, $n$, to a desired value.

It is verified with simulation and measurement results that the anti-resonance peak can be separated from the mechanical resonance by increasing the equivalent capacitance of the gyroscope in the lumped model or by decreasing the stray capacitances. As the stray capacitances are limited by hybrid wirebonding, we prefer increasing the gyroscope equivalent capacitance by tuning the electromechanical conversion parameter, $n$, in Eq. (5). Fig. 17 shows the simulation results when the gyroscope equivalent capacitance in the lumped model is increased. This can be achieved by either lowering the spring constants (in the design phase), or increasing the sensor capacitance (in the design phase or improving the process), or increasing the dc polarization voltage. Fig. 18 shows the measured resonance characteristics of the gyroscope when the dc polarization voltage of the gyroscope is increased up to $100 \mathrm{~V}$, which provide a much clear magnitude and phase characteristics at resonance.

The measurement results show that the fabricated gyroscopes have slightly mismatched resonance frequencies for the drive and sense modes due to manufacturing tolerances, even though a symmetric design is utilized. The resonance frequencies can be slightly tuned by changing the dc polarization voltage applied to the proof mass. Fig. 19 shows the frequency shift characteristics for the drive and sense resonances as a function of dc polarization voltages. Due to the nature of the linear comb drives, the amount of frequency shift to tune the drive and sense mode resonances requires higher dc tuning voltages, limited by the breakdown volt- ages associated with the narrow electrostatic gaps and glass substrate. It is possible to achieve larger tuning range with reduced dc voltages by using additional electrostatic tuning electrodes in future designs.

The gyroscope is also tested under $0.04 \mathrm{mbar}$ vacuum ambient, to check any improvements in the mechanical quality factors at resonance. Fig. 20 shows the drive-mode resonance characteristics both at atmospheric pressure and at vacuum ambient, yielding an improvement in the quality factor from 200 to 300 only, meaning that the thermoelastic damping is still dominant in the fabricated nickel microgyroscopes. However, an advantage of vacuum operation is that the amount of parasitic signal coupling is decreased to a great extent, due to decreased actuation signal level.

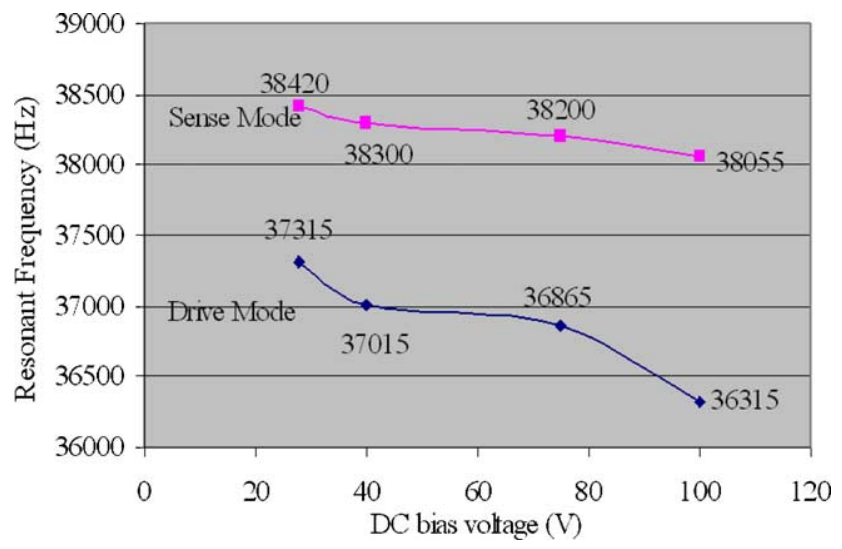

Fig. 19. Frequency shift characteristics for the drive and sense resonances as a function of dc polarization voltages. 

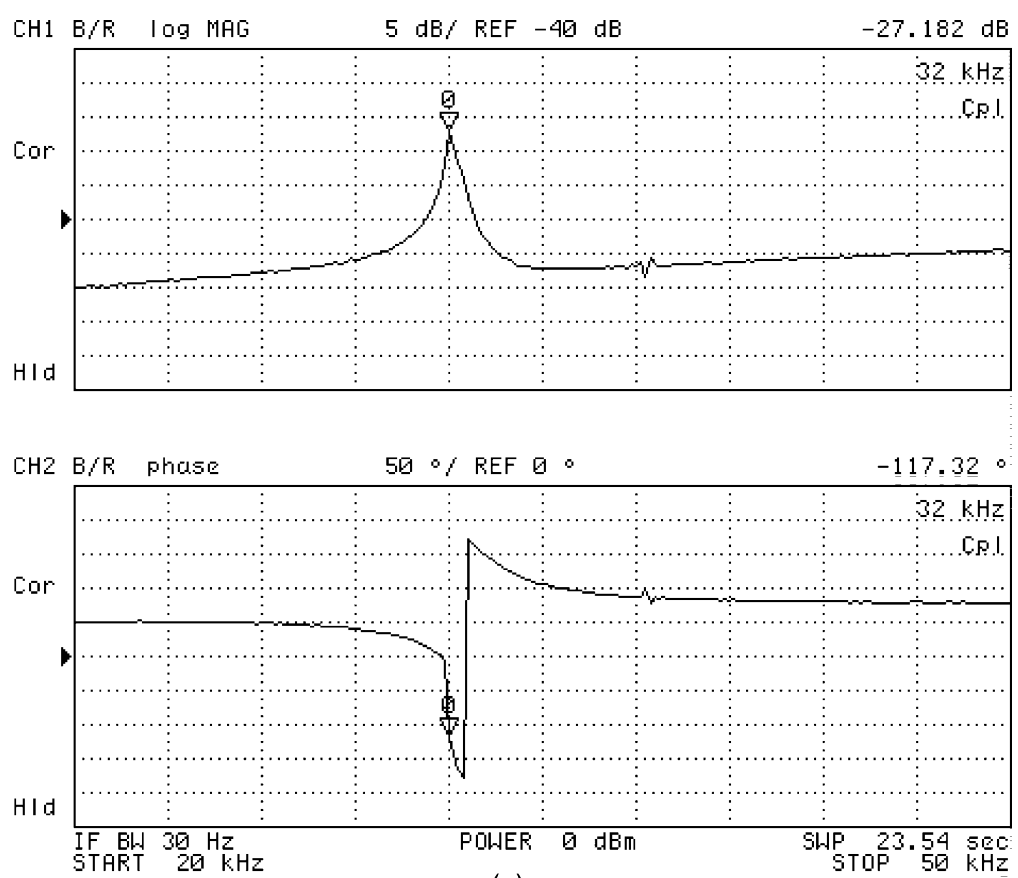

(a)
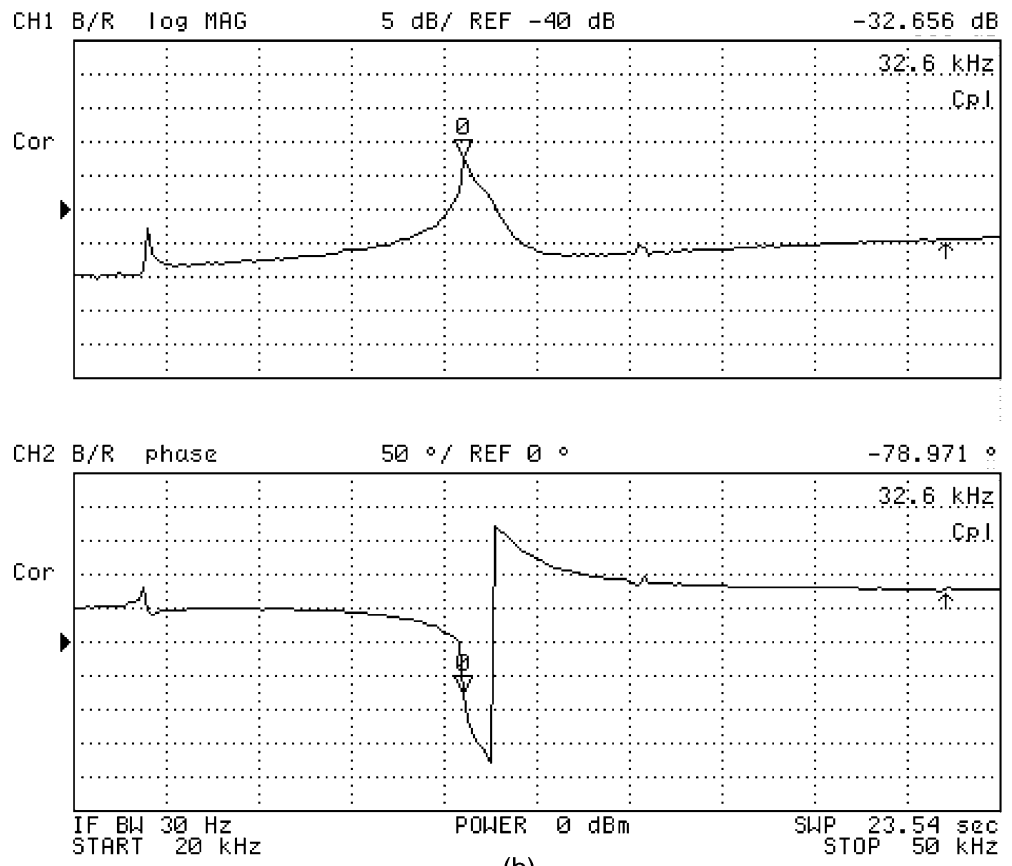

(b)

Fig. 20. Drive-mode resonance characteristics both at (a) atmospheric pressure and (b) 0.04 mbar vacuum ambient.

The overall performance of the fabricated gyroscope can be determined using the measurement results and theoretical thermomechanical noise floor of the gyroscope. The thermomechanical noise of the fabricated gyroscope is determined by the following equation:

$$
\bar{\Omega}(k)_{\text {Brownian }}^{2}=\frac{2 k_{\mathrm{B}} T w_{\text {sense }}}{m Q_{\text {sense }} X_{0}^{2} w_{\text {drive }}^{2}}, \quad Q_{\text {sense }}=\frac{\sqrt{k_{\text {sense }} m}}{b_{\text {sense }}}
$$

where $k_{\mathrm{B}}$ is the Boltzmann constant, $T$ the temperature, $Q_{\text {sense }}$ the mechanical quality factor of the sense mode, and $X_{0}$ is the drive mode vibration amplitude. The fabricated gyroscope with a $Q_{\text {sense }}$ of 500 provides a Brownian noise floor of $7.3^{\circ} / \mathrm{h} / \mathrm{Hz}^{1 / 2}$ at vacuum. Currently, the sensitivity of the gyroscope is limited to $96^{\circ} / \mathrm{h}$ in $50 \mathrm{~Hz}$ bandwidth for matched-frequency operation, due to low sense mode quality factor at vacuum. Improving the quality of the electrodeposited nickel, the overall rate sensitivity of the gyroscope 
is estimated to reach to $56^{\circ} / \mathrm{h}$ in $50 \mathrm{~Hz}$ bandwidth including both the mechanical and electronic noise floors, provided that the gyroscope is operated at vacuum and that the resonance frequencies of the drive and sense modes are closely matched.

\section{Conclusions and future work}

This paper presents a symmetrical and decoupled surface micromachined gyroscope fabricated by electroforming thick nickel on a glass substrate. The symmetric structure allows matched resonant frequencies for the drive and sense vibration modes for improved sensitivity, while the decoupled drive and sense oscillation modes prevents unstable operation due to mechanical coupling and achieving a low zero-rate output drift. The use of a glass substrate instead of a silicon substrate reduces noise due to the parasitic signal coupling by two orders of magnitude according to both simulated and measured results on fabricated devices. The fabricated gyroscope is hybrid connected to a capacitive interface circuit which is fabricated in a $0.8 \mu \mathrm{m}$ CMOS process, where the circuit has an input capacitance lower than $50 \mathrm{fF}$ and a sensitivity of $33 \mathrm{mV} / \mathrm{fF}$. Fabricated gyroscopes have close resonant frequencies for the drive and sense modes, as 37.2 and $38.3 \mathrm{kHz}$, respectively, and can be further tuned by changing dc polarization voltage applied to the proof mass. The fabricated gyroscope with $16 \mu \mathrm{m}$-thick structural layer provides a Brownian noise floor of $7.3^{\circ} / \mathrm{h} / \mathrm{Hz}^{1 / 2}$ at vacuum. Currently, the overall rate sensitivity of the gyroscope is limited to $96^{\circ} / \mathrm{h}$ in $50 \mathrm{~Hz}$ bandwidth for matched-frequency operation, and it can be decreased down to $56^{\circ} / \mathrm{h}$ in $50 \mathrm{~Hz}$ bandwidth, by improving the quality of the electrodeposited nickel.

\section{Acknowledgements}

This work is sponsored by the National Defense Ministry (MSB ArGe), State Planning Organization (DPT), and The Scientific and Technical Research Council of Turkey (TUBITAK EEEAG-100E020).

\section{References}

[1] N. Yazdi, F. Ayazi, K. Najafi, Micromachined inertial sensors, Proc. IEEE 86 (8) (1998) 1640-1659.

[2] Y. Mochida, M. Tamura, K. Ohwada, A micromachined vibrating rate gyroscope with independent beams for the drive and detection modes, in: Proceedings of the IEEE Micro-Electro-Mechanical Systems Workshop (MEMS'99), January 1999, pp. 618-623.

[3] W. Geiger, J. Merz, T. Fischer, B. Folkmer, H. Sandmaier, W. Lang, The silicon angular rate sensor system MARS-RR, in: Proceedings of the 10th International Conference Solid-State Sensors and Actuators (Transducers'99), June 1999, pp. 1578-1581.

[4] M.S. Kranz, G.K. Fedder, Micromechanical vibratory rate gyroscopes fabricated in conventional CMOS, in: Proceedings of the Symposium on Gyro Tech., 1997, pp. 3.0-3.8.
[5] S.E. Alper, T. Akin, A symmetric surface micromachined gyroscope with decoupled oscillation modes, Sens. Actuators A 97C/98C (2002) 347-358.

[6] S.E. Alper, T. Akin, A symmetrical and decoupled microgyroscope with electroforming process on insulating substrate, in: Proceedings of the 16th European Conference on Solid-State Transducers (Eurosensors XVI), September 2002, pp. 814-817.

[7] S.E. Alper, T. Akin, A symmetrical and decoupled nickel microgyroscope on insulating substrate, in: Proceedings of the 17th European Conference on Solid-State Transducers (Eurosensors XVII), September 2003, pp. 24-27.

[8] S.D. Senturia, Microsystem Design, Kluwer Academic Publishers, 2001, Chapter 21, pp. 561-604.

[9] C.T.C. Nguyen, Micromechanical signal processors, Ph.D. Dissertation, University of California, Berkeley, 1994.

[10] W.A. Clark, R.T. Howe, Surface micromachined $z$-axis vibratory rate gyroscope, in: Proceedings of the Solid-State Sensor and Actuator Workshop, 1996, pp. 283-287.

[11] G.K. Fedder, Simulation of microelectromechanical systems, Ph.D. Dissertation, University of California, Berkeley, 1994.

[12] B.E. Boser, Electronics for micromachined inertial sensors, in: Proceedings of the Ninth International Conference on Solid-State Sensors and Actuators (Transducers'97), June 1997, pp. 1169-1172.

\section{Biographies}

Said Emre Alper was born in Ankara, Turkey, in 1976. He received the BS and MSc degrees in electrical and electronics engineering with high honors from Middle East Technical University (METU) in Ankara, in 1998 and 2000, respectively. He is currently working towards the PhD degree in electrical and electronics engineering at METU. Since 1998, he has been working as a research assistant at METU in the Department of Electrical and Electronics Engineering, MEMS VLSI Research Group. His major research interests include capacitive inertial sensors, micromachined resonators and actuators, capacitive interface circuits, and microfabrication technologies. He is the first author of the symmetric and decoupled gyroscope design, which won the first prize award in the operational designs category of the "International Design Contest" organized by DATE and CMP in March 2001. He is also the first author of the tactical-grade symmetrical and decoupled micro-gyroscope design, which won the third prize award in the international "3-D MEMS Design Challenge" organized by MEMGEN Corp. (currently Microfabrica), in June 2003, among 132 MEMS designs from 24 countries and 25 states across the US.

Tayfun Akin was born in Van, Turkey, in 1966. He received the BS degree in electrical engineering with high honors from Middle East Technical University, Ankara, in 1987 and went to the US in 1987 for his graduate studies with a graduate fellowship provided by NATO Science Scholarship Program through the Scientific and Technical Research Council of Turkey (TUBITAK). He received the MS degree in 1989 and the PhD degree in 1994 in electrical engineering, both from the University of Michigan, Ann Arbor. Since 1995, 1998 and 2004, he has been employed as an assistant professor, associate professor and professor, respectively, in the Department of Electrical and Electronics Engineering at Middle East Technical University, Ankara, Turkey. He is also the technical coordinator of METU-MET, an IC fabrication factory which is transferred to Middle East Technical University by the government for MEMS related production. His research interests include MEMS (Micro-Electro-Mechanical Systems), microsystems technologies, infrared detectors and readout circuits, silicon-based integrated sensors and transducers, and analog and digital integrated circuit design. He has served in various MEMS, EUROSENSORS, and TRANSDUCERS conferences as a technical program committee member. He is the designate co-chair of The 19th IEEE International Conference of Micro-Electro-Mechanical Systems (MEMS 2006) 
to be held in Istanbul. He is the winner of the first prize in experienced analog/digital mixed-signal design category at the 1994 Student VLSI Circuit Design Contest organized and sponsored by Mentor Graphics, Texas Instruments, Hewlett-Packard, Sun Microsystems, and Electronic Design Magazine. He is the co-author of the symmetric and decoupled gyroscope project which won the first prize award in the operational designs category of the international design contest organized by DATE conference and CMP in March 2001. He is also the co-author of the gyroscope project which won the third prize award of "3-D MEMS Design Challenge" organized by MEMGEN Corp. (currently Microfabrica). 